\section{The problem of ignoring interconnectedness in genetic research}

SIR

I was delighted to read a scholarly defence of the notions of interconnectedness and responsibility to others as they apply to genetic privacy. ${ }^{1}$ Many of the same ethical principles that apply to providing medical care also apply to conducting research.

Concepts of genetic privacy are evolving, and these concepts can have a profound effect on the conduct of genetic and genealogical research. In the United States, there seems to be an emphasis on autonomy to the near exclusion of responsibility to others in human genetic research. A recent case illustrates the point. ${ }^{2}$ The father of college-age twins had complained that a questionnaire sent to his daughter by a university geneticist invaded his privacy by asking questions about his own physical and emotional health. The issue was raised to the level of the National Institutes of Health (US), and the Office for the Protection from Research Risk (OPRR) responded by temporarily suspending federal funding of research at the university. The OPRR said the investigator should have secured consent from each family member included in the pedigree. Operationally, the OPRR was treating each member of a pedigree as an unrelated autonomous individual.

The temporary suspension of funding had a chilling effect on review of genetic research protocols at many institutions. My experience with planning a study that involves many clinical centres in North America has taught me that many institutional review boards fear that contacting family members using information freely given by probands might be construed as infringing the privacy rights of family members. Cold contacting is the process of contacting a stranger without securing permission. To the consternation of many in the United States, the process is done routinely by telemarketers. Should cold contacting be banned in genetic research? Naturally, people have the right to refuse participation in a research study. It also seems appropriate that participation of a family member in a research protocol should in no way restrict the right of another member of that same pedigree to refuse participation in a research study. Cold contacting to invite additional members of a pedigree to participate in a genetic study needs to be reconsidered in communitarian terms. Notions of interconnectedness and responsibility to others are atrophied and are in need of exercise.

\section{References}

1 Sommerville A, English V. Genetic privacy: orthodoxy or oxymoron? fournal vacy: orthodoxy or oxymoron? fourn
of Medical Ethics 1999;25:144-50.

2 Wadman M. US geneticists encour-

aged to play by the book. Nature 2000; 404:530.

JAMES F MESCHIA, MD Consultant and Assistant Professor of Neurology, Mayo Clinic, 4500 San Pablo Road Facksonville, Florida 32224, USA

\section{Single blind placebo in drug research}

SIR

The recent article by Evans in the journal on the single blind placebo in drug research is timely and its conclusions were persuasive. The basic premiss that single blind placebo "washout" periods are ethically specious was well argued and I agree that from a scientific point of view they have no valid justification. The real reasons for attempting to blind these portions of clinical trials were defined very completely by Evans and scientifically the studies would not be undermined by merely taking the patients off active treatment altogether.

There is, however, one area not mentioned by Evans where I think that a single blind trial is not only justified but is in fact ethically and scientifically more honest. This could occur when in a placebo controlled study a drug under trial has such obvious clinical effects that to a trained observer it would be immediately apparent which patients were on active treatment. The early trials of beta-blockers versus placebo would be an obvious example where the slow pulse rate produced by the active drug would render double blind a spurious designation. Similarly I was involved in a drug trial many years ago comparing a long-acting anticholinergic preparation with placebo in patients with peptic ulcer disease. The active drug produced such obvious side effects of dry mouth, blurred vision, etc, that a purposeful decision was taken to call this a single blind, rather than a double blind study, on the grounds that the investigators would very easily be able to tell who was on active treatment and who was not.

Clearly this is a very different situation from the use of washout placebos but I feel that the very contrast is supportive of Evans's case.

\section{References}

1 Evans M. Justified deception? The single blind placebo in drug research. Fournal of Medical Ethics 2000;26:18893.

2 Kaye MD, Beck P, Rhodes J, Sweetman PM. Gastric acid secretion in patients with duodenal ulcer treated for patients with duodenal ulcer treated for
one year with anti-cholinergic drugs. Gut 1969;10:774.

PETER BECK

Consultant Physician, Llandough Hospital, Penlan Road, Llandough, Penarth Vale of Glamorgan CF54 2XX 